\title{
Effects of soft tissue on the crystallographic changes to bone mineral upon heating
}

\author{
H. Cross ${ }^{1}$, C. Greenwood ${ }^{1}$ \\ ${ }^{1}$ Keele University, Keele, Staffordshire, ST5 5BG, United Kingdom \\ h.l.cross@keele.ac.uk,c.e.greenwood@keele.ac.uk
}

Upon the recovery of burnt remains in a forensic or archaeological context, bone is often fragmented and comingled, making differentiation between human and non-human samples extremely challenging and subjective. Due to thermal degradation of the organic component, biological techniques, such as DNA analysis, often render futile and so attention is drawn to the final surviving component of bone, the mineral hydroxyapatite.

Exploring the physicochemical modifications that occurs to hydroxyaptite upon heating has shown promise in differentiating between species based on characteristic changes within its crystal lattice structure, and the presence of extraneous mineral phases [1]. However, the effects soft tissue has on the heat induced changes are not fully understood, yet are of paramount importance as most bodies are intact, not skeletonised, during a burning event. This study aims to explore the effect heating has on fleshed bone, specifically investigating modifications to the nanocrystalline structure of bone mineral, and whether this has a significant impact on species differentiation.

Varying weights $(5 \mathrm{~g}, 7 \mathrm{~g}$ and $10 \mathrm{~g})$ of muscle and fat, and one layer of skin were tested separately to understand their individual affect. A combination of the three tissue types was also considered. The samples were heated for two hours at various temperatures $\left(200^{\circ} \mathrm{C}\right.$, $400^{\circ} \mathrm{C}, 600^{\circ} \mathrm{C}$ and $900^{\circ} \mathrm{C}$ ) which are representative of those temperatures reached in historical forensic and archaeological cases. Powder X-ray diffraction (pXRD) analysis was utilised to calculate coherence length and lattice parameter values and the weight percentages of extraneous mineral phases to identify species differentiating characteristics. Coherence length, which gives an indication on crystallite size and strain, was calculated using the Scherrer equation and the full width half maximum (FWHM) peak values. Spectroscopic techniques including Fourier Transform Infrared (FTIR) and Raman spectroscopy were utilised to collaborate the XRD data and to further our understanding of the relationship between the degradation of the organic matrix and the crystallographic changes.

[1] Beckett, S., Rogers, K. D., Clement, J. G. (2011). J Forensic Sci, 3, 571-579.

Keywords: Heated bone; X-ray diffraction; forensic science; fleshed bone; crystallographic parameters. 